\title{
THE PERFECTION AND RECOGNITION OF BULL-REDUCIBLE BERGE GRAPHS *,**
}

\author{
Hazel Everett ${ }^{1}$, Celina M.H. De Figueiredo ${ }^{2}$, \\ Sulamita Klein ${ }^{2}$ and Bruce Reed ${ }^{3}$
}

\begin{abstract}
The recently announced Strong Perfect Graph Theorem states that the class of perfect graphs coincides with the class of graphs containing no induced odd cycle of length at least 5 or the complement of such a cycle. A graph in this second class is called Berge. A bull is a graph with five vertices $x, a, b, c, d$ and five edges $x a, x b, a b, a d, b c$. A graph is bull-reducible if no vertex is in two bulls. In this paper we give a simple proof that every bull-reducible Berge graph is perfect. Although this result follows directly from the Strong Perfect Graph Theorem, our proof leads to a recognition algorithm for this new class of perfect graphs whose complexity, $O\left(n^{6}\right)$, is much lower than that announced for perfect graphs.
\end{abstract}

Mathematics Subject Classification. 05C17, 05C75, $05 \mathrm{C} 85$.

\section{INTRODUCTION}

A graph is perfect if for every induced subgraph $F$ of $G$ the chromatic number $\chi(F)$ of $F$ is equal to its clique number $\omega(F)$ (i.e., the size of the largest clique contained in $F$ ). It is possible to color a perfect graph optimally and in polynomial time, thanks to the algorithm of Grötschel, Lovász and Schrijver [9], but that algorithm, based on the ellipsoid method, is rather impractical. Polynomialtime combinatorial algorithms, based on a structural analysis and decomposition of perfect graphs are still being sought. Lovász's Perfect Graph Theorem says that the class of perfect graphs is self-complementary [14]. A hole is an induced cycle

* Part of this research was done while the third author was visiting the Equipe Combinatoire, Université Pierre et Marie Curie, Paris 6, supported by CAPES.

** Partially supported by CNPq, PRONEX 107/97 and CAPES/COFECUB.

${ }^{1}$ LORIA, France; Hazel.Everett@loria.fr

2 Universidade Federal do Rio de Janeiro, Brasil; \{celina, sula\}@cos.ufrj.br

3 McGill University, Canada; breed@cs.mcgill.ca 


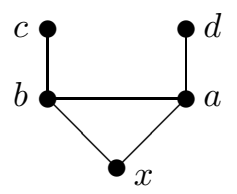

Figure 1. The bull $\mathcal{B}(c, b x a, d)$.

of length $\geq 5$. Berge's well known Strong Perfect Graph Conjecture states that the class of perfect graphs coincides with the class of graphs containing no odd hole or the complement of an odd hole. A graph in this second class is called Berge. It is easy to see that every perfect graph is Berge so the Strong Perfect Graph Conjecture actually states that every Berge graph is perfect. For partial results on this conjecture see $[1,15]$. The Strong Perfect Graph Conjecture is considered a central problem in computational complexity, combinatorial optimization, and graph theory.

In October 2002, Chudnovsky, Seymour, Robertson and Thomas announced a proof of the Strong Perfect Graph Conjecture. The preliminary version of their paper, consisting of 148 pages [4], is long and complex. The proposed decomposition theorem that proves the Strong Perfect Graph Conjecture does not lead to an efficient recognition algorithm, nor does it immediately imply that the four optimization problems related to the definition of perfect graphs (maximum clique, minimum colouring, maximum stable set, minimum clique cover) can be solved efficiently for perfect graphs. Subsequently, polynomial-time algorithms to recognize Berge graphs have been announced by Chudnovsky, Cornuejols, Liu, Seymour and Vuskovic $[3,5,13]$; these algorithms have high complexities, $O\left(n^{9}\right)$, and $O\left(n^{20}\right)$. Polynomial-time combinatorial algorithms for the four optimization problems, based on a structural analysis and decomposition of perfect graphs are still being sought.

The purpose of the present paper is to give a simple proof for the validity of the Strong Perfect Graph Conjecture for a subclass of Berge Graphs. The proof of the Strong Perfect Graph Conjecture proposed in [4] claims that every Berge Graph can be decomposed by 2-join, M-join or skew partition decomposition into basic perfect graphs: bipartite or complement of bipartite, line graph of bipartite or double split. In this paper, we prove Theorem 3, a statement for bull-reducible graphs stronger than the direct application of [4]. In addition, our proof of the Strong Perfect Graph Conjecture for a subclass of Berge Graphs yields a polynomial-time algorithm to recognize the corresponding subclass of perfect graphs in time $O\left(n^{6}\right)$.

Denote by $\mathcal{B}(c, b x a, d)$ the labelled graph depicted in Figure 1, a bull on five vertices $x, a, b, c, d$ and five edges $x a, x b, a b, a d, b c$. Vertex $x$ is the nose of this bull. Note that if $x$ is the nose of a bull $\mathcal{B}(c, b x a, d)$ in $G$, then $x$ is the nose of a bull $\mathcal{B}(a, c x d, b)$ in $\bar{G}$. The class of bull-free graphs is self-complementary and generalizes the class of $P_{4}$-free graphs. 
Chvátal and Sbihi $[6]$ proved:

Theorem 1. Every bull-free Berge graph is perfect.

Since then others have studied bull-free Berge graphs; see [16] for a polynomialtime recognition algorithm; $[7,8]$ for an alternative proof of perfection, and a bipartite layout of bull-free Berge graphs; [11] for a proof of a conjecture of Chvátal.

We extend the result of Theorem 1 and prove that if no vertex in a Berge graph $G$ is in two bulls then $G$ is perfect. We also provide a polynomial recognition algorithm for such graphs, which we call bull-reducible Berge graphs (following the definition of $P_{4}$-reducible in Jamison and Olariu [12]).

The main tool we use is the star cutset. A star cutset in $G$ is a vertex cutset $X$ such that for some vertex $x$ of $X, X \subseteq x \cup N(x)$, where $N(x)$ is the neighbourhood of $x$. Note that $N(x)$ does not include $x$. We say that vertex $x$ sees vertex $y$, if $y \in N(x)$, and that vertex $x$ misses vertex $y$, if $y \notin N(x)$.

Chvátal [2] proved:

Theorem 2. No minimal imperfect graph contains a star cutset.

In this paper, we prove:

Theorem 3. If $G$ is a bull-reducible $C_{5}$-free graph which is not bull-free, then $G$ or $\bar{G}$ contains a star cutset.

Combining our Theorem 3 with Theorems 1 and 2 yields:

Theorem 4. Every bull-reducible Berge graph is perfect.

Sbihi and Reed [16] presented a polynomial-time recognition algorithm for bullfree Berge graphs. In this paper, we propose a polynomial-time algorithm that reduces the recognition of bull-reducible Berge graphs to the recognition of bullfree Berge graphs.

\section{Neighbourhood OF A HOLE}

Call a graph unbreakable if neither $G$ or $\bar{G}$ has a star cutset. The key to our proof of Theorem 3 is an analysis of the possible intersections of the neigbourhood of a vertex with a hole in an unbreakable bull-reducible graph.

Lemma 1 (domination lemma). If $G$ is a bull-reducible, $C_{5}$-free graph and $\mathcal{B}(c, b x a, d)$ is a bull in $G$, then $N(x) \subset N(a) \cup N(b)$.

Proof. Let $y \in N(x)$. Suppose $y \notin N(a) \cup N(b)$. If $y$ sees both $c$ and $d$, then $\{y, c, b, a, d\}$ induces a $C_{5}$. Otherwise $y$ misses $c$ or $d$. If $y$ misses $c$, then $\mathcal{B}(c$, bax, $y)$ induces a bull, which contradicts $G$ being bull-reducible. Similarly, if $y$ misses $d$. Therefore $y \in N(a) \cup N(b)$. 


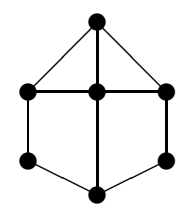

Figure 2. $N(v) \cap H$ consists of exactly four vertices.

Lemma 2 (neighbourhood lemma). Let $G$ be a bull-reducible, $C_{5}$-free graph. Let $H$ be a shortest hole in $G$. Let $v$ be a vertex of $G$ not in $H$. The neighbourhood $N(v) \cap H$ of $v$ with respect to $H$ satisfies one of the five properties:

(i) $N(v) \cap H$ is a stable set;

(ii) $N(v) \cap H$ is an edge;

(iii) $N(v) \cap H$ consists of exactly three consecutive vertices of $H$;

(iv) $N(v) \cap H$ consists of exactly four vertices of $H,|H|=6$, and $v$ plus the vertices of $H$ induce the graph in Figure 2;

(v) $N(v) \cap H$ consists of all vertices of $H$.

Proof. Let the vertices of the hole $H$ be labelled $v_{1}, v_{2}, \ldots, v_{n}$, such that $v_{i}$ is adjacent to $v_{i+1}$, with indices taken modulo $n$, and $n=|H|$. Suppose first that $N(v) \cap H$ is not a stable set. Hence, $N(v) \cap H$ contains at least two consecutive vertices (i.e., an edge) of $H$. Suppose $N(v) \cap H$ contains two consecutive vertices $v_{1}, v_{2}$, and a third vertex $c$.

Claim 2.1. $N(v) \cap H$ contains three consecutive vertices.

Proof. Otherwise, let $v_{n}, v_{1}, v_{2}, v_{3}$ be consecutive in $H$ and suppose that $v$ misses both $v_{n}$ and $v_{3}$. Note that $\mathcal{B}\left(v_{n}, v_{1} v v_{2}, v_{3}\right)$ is a bull. Since $|H| \geq 6$, we have that $c$, a third vertex seen by $v$, misses $v_{n}$ or $v_{3}$. Suppose $c$ does not see $v_{n}$. Now $\mathcal{B}\left(v_{n}, v_{1} v_{2} v, c\right)$ is a bull intersecting $\mathcal{B}\left(v_{n}, v_{1} v v_{2}, v_{3}\right)$, a contradiction.

Claim 2.2. If $N(v) \cap H$ contains four consecutive vertices, then $N(v) \cap H$ consists of all vertices of $H$.

Proof. Suppose $v$ is adjacent to $v_{1}, v_{2}, \ldots, v_{j}, 4 \leq j<n$, but $v$ is not adjacent to $v_{j+1}$ and $v_{n}$. Now $\mathcal{B}\left(v_{n}, v_{1} v_{2} v, v_{4}\right)$ and $\mathcal{B}\left(v_{j-3}, v v_{j-1} v_{j}, v_{j+1}\right)$ are two distinct bulls containing $v$.

It remains to show that in case $v$ sees at least three consecutive but not four consecutive vertices, and $v$ sees at least one more vertex in $H$, then $N(v) \cap H$ consists of four vertices, $|H|=6$, and $v$ plus the vertices of $H$ induce the graph in Figure 2.

In order to establish that, assume that $v$ sees $v_{1}, v_{2}, v_{3}$, but $v$ does not see $v_{n}, v_{4}$, and $v$ sees $v_{j}$, with $j \neq 1,2,3,4, n$.

Let $i$ be the smallest such index and let $k$ be the largest such index. Since $H$ is a shortest hole in $G$, we have $i=5$ and $k=n-1$. Now, if $n>6$, then $\mathcal{B}\left(v_{n}, v_{1} v_{2} v, v_{5}\right)$ and $\mathcal{B}\left(v_{n-1}, v v_{2} v_{3}, v_{4}\right)$ induce two distinct bulls containing $v$. 
We say that a graph $G$ contains a homogeneous set $O$, if the vertex set $V$ of $G$ admits a partition into three subsets $O, A$ and $N$, such that $O$ contains at least two vertices, $A \cup N$ is nonempty, we have all edges between $O$ and $A$, and we have no edges between $O$ and $N$. The concept of star cutset generalizes the concept of homogeneous set in the following way:

Fact 1. If $G$ contains a homogeneous set, then $G$ or $\bar{G}$ contains a star cutset.

Lemma 3 (wheel lemma). Let $G$ be a bull-reducible, $C_{5}$-free graph, and $x$ be a vertex of $G$. If $N(x)$ contains a hole of size at least 6 , then $G$ contains a homogeneous set.

Proof. Suppose $N(x)$ contains a hole $H,|H| \geq 6$. Let the vertices of the hole $H$ be labelled $v_{1}, v_{2}, \ldots, v_{n}$, such that $v_{i}$ is adjacent to $v_{i+1}$, with indices taken modulo $n$, and $n=|H|$. Let $A$ be the set of vertices that see all of $H$. By hypothesis, $A$ is nonempty since it contains vertex $x$. Let $O$ be the connected component of $G-A$ that contains $H$.

We shall prove that $O$ is a homogeneous set of $G$. It is enough to prove that every vertex in $A$ is adjacent to every vertex of $O$. Let $a \in A, h \in O$. We argue by induction on $d(h, H)$, the distance of $h$ to the hole $H$.

Case 3.1. $d(h, H)=0$.

If $d(h, H)=0$, then $h \in H$ and the definition of $A$ implies that $a h \in E$.

Case 3.2. $d(h, H)=1$.

Assume $h a \notin E$. Without loss of generality, we assume that $h$ sees vertex $v_{1}$. Suppose $h$ does not see any edge of $H$, i.e., $h$ does not see two consecutive vertices of $H$. Now $h$ has to see vertex $v_{4}$, as otherwise $\mathcal{B}\left(v_{4}, a v_{2} v_{1}, h\right)$ and $\mathcal{B}\left(v_{4}, a v_{n} v_{1}, h\right)$ are intersecting bulls. But this implies that $h$ misses $v_{3}$ and $v_{5}$, and now $\mathcal{B}\left(h, v_{4} v_{5} a, v_{2}\right)$ and $\mathcal{B}\left(h, v_{4} v_{3} a, v_{n}\right)$ are intersecting bulls. Therefore $h$ does see at least one edge of $H$.

Suppose $h$ sees just the edge $v_{1} v_{2}$. Then we have intersecting bulls $\mathcal{B}\left(v_{n}, v_{1} h v_{2}, v_{3}\right)$ and $\mathcal{B}\left(h, v_{2} v_{3} a, v_{5}\right)$.

So, by the proof of Claim 2.1 of Lemma 2 (neighbourhood lemma), $h$ sees at least 3 consecutive vertices of $H$, say $v_{1}, v_{2}, v_{3}$. Note that $h$ cannot see $v_{4}$ or $v_{n}$, as this, by the proof of Claim 2.2 of Lemma 2 would imply $h \in A$, a contradiction to our hypothesis. But now we have two intersecting bulls $\mathcal{B}\left(h, v_{3} v_{4} a, v_{n}\right)$ and $\mathcal{B}\left(h, v_{1} v_{n} a, v_{4}\right)$, implying $a h \in E$.

Case 3.3. $d(h, H)=2$.

Let $P=h u v_{1}$ be a shortest path joining $h$ to $H$. By induction, we have $a u \in E$.

Suppose $|H|>6$. In this case, we can find $z, z^{\prime} \in H-\left\{v_{1}, v_{2}, v_{n}\right\}$ such that $z u, z^{\prime} u \notin E$. Now $\mathcal{B}\left(h, u v_{1} a, z\right), \mathcal{B}\left(h, u v_{1} a, z^{\prime}\right)$ are intersecting bulls, implying $a h \in E$.

Suppose $|H|=6$. In addition, suppose we cannot find $z, z^{\prime} \in H-\left\{v_{1}, v_{2}, v_{6}\right\}$ such that $z u, z^{\prime} u \notin E$. The proof of Lemma 2 says that $u$ sees $v_{3}, v_{5}$, besides $v_{1}$, and $u$ does not see $v_{2}$ and $v_{6}$. But now we have intersecting bulls $\mathcal{B}\left(h, u v_{3} a, v_{6}\right)$ and $\mathcal{B}\left(h, u v_{5} a, v_{2}\right)$, implying $a h \in E$. 


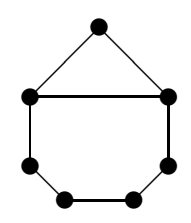

Figure 3. A cap.

Case 3.4. $d(h, H) \geq 3$.

Let $P=h u w_{1} w_{2} \ldots w_{k}$ be a shortest path joining $h$ to $H$, i.e., $k \geq 2 ; w_{k} \in H$; and $h, u, w_{1}, \ldots, w_{k-1} \notin H$. Let $z, z^{\prime}$ be two vertices missed by $w_{1}$ in $H$. We have that $z, z^{\prime}$ are also missed by $u$, as $d(h, H) \geq 2$. By induction, we have $a u, a w_{1} \in E$. Hence we have two intersecting bulls: $\mathcal{B}\left(z, a w_{1} u, h\right)$ and $\mathcal{B}\left(z^{\prime}, a w_{1} u, h\right)$, implying $a h \in E$.

This ends the proof of Lemma 3 .

A cap is a cycle on at least seven vertices having a single chord that forms a triangle with two edges of the cycle. (See Fig. 3.)

Lemma 4 (cap lemma). Let $G$ be bull-reducible, unbreakable, $C_{5}$-free. Then $G$ contains no cap.

Proof. Suppose that $G$ contains a cap. Let $H$ be a hole of $G$. Let the vertices of the hole $H$ be labelled $v_{1}, v_{2}, \ldots, v_{n}$, such that $v_{i}$ is adjacent to $v_{i+1}$, with indices taken modulo $n$, and $n=|H|$. Call vertices $v_{1}, v_{2}, v_{3}$ and $v_{n}$ by $a, b, c$ and $d$, respectively. Let $x$ be a vertex of $G-H$ such that $N(x) \cap H$ is the edge $a b$ of $H$ that is, $x$ and $H$ form a cap. Note that the hypothesis implies the bull $\mathcal{B}(d, a x b, c)$. By Lemma 1 (domination lemma), $N(x) \subset N(a) \cup N(b)$.

Claim 4.1. $N(x) \cap N(a) \cap N(b)=\emptyset$.

Proof. Let $t$ be a vertex in $N(x) \cap N(a) \cap N(b)$. Now, the bull $\mathcal{B}(d, a t b, c)$ implies $t d$ or $t c$. Vertex $t$ cannot see both $d$ and $c$, as otherwise by the proof of Claim 2.2 of Lemma 2 (neighbourhood lemma), $t$ sees all of $H$ and by Lemma 3 (Wheel Lemma) we have a contradiction to $G$ being unbreakable. Now, if $t$ is adjacent to only one vertex in $\{d, c\}$, say we have edge $t d$, we have the bull $\mathcal{B}(d, t x b, c)$.

Hence $N(x)$ can be partitioned into $A=N(x) \cap N(a)$ and $B=N(x) \cap N(b)$.

Claim 4.2. Every $t \in A=N(x) \cap N(a)$ sees every $z \in B=N(x) \cap N(b)$.

Proof. Let $t \in A-\{b\}$, and $z \in B-\{a\}$. Suppose $t$ misses $z$. If $z$ sees $c=v_{3}$, then $z$ sees $v_{4}$ as otherwise $\mathcal{B}\left(a, b z c, v_{4}\right)$ is a bull. Now $z$ does not see $v_{5}$, as otherwise $z$ sees all of $H$. Now $\mathcal{B}\left(x, z c v_{4}, v_{5}\right)$ is a bull. Hence $z$ misses $c$ and by symmetry $t$ misses $d$. Now $t$ sees $c$, as otherwise $\mathcal{B}(t, x z b, c)$ is a bull. And by symmetry, $z$ sees $d$. Finally, the bull $\mathcal{B}(c, \operatorname{tax}, z)$ implies edge $t z$. 
Now $x \cup \overline{N(x)}$ is a star cutset in $\bar{G}$ separating $A$ and $B$. This ends the proof of Lemma 4.

We are ready to state a revised version of Lemma 2. Note that in Lemma 5 the hypothesis of $H$ being shortest is not required.

Lemma 5 (neighbourhood lemma revisited). Let $G$ be a bull-reducible, $C_{5}$-free, unbreakable graph. Let $H$ be a hole in $G$. Let $v$ be a vertex of $G$ not in $H$. The neighbourhood $N(v) \cap H$ of $v$ with respect to $H$ satisfies one of the three properties:

(i) $N(v) \cap H$ is a stable set;

(ii) $N(v) \cap H$ consists of exactly three consecutive vertices of $H$;

(iii) $N(v) \cap H$ consists of exactly four vertices of $H,|H|=6$, and $v$ plus the vertices of $H$ induce the graph in Figure 2.

Proof. Apply Lemma 2, Fact 1, Lemmas 3 and 4.

\section{Proof of Theorem 3}

To prove Theorem 3, we consider a graph $G$ which is $C_{5}$-free, bull-reducible but not bull-free and let $x$ be the nose of some bull of $G$. We suppose further that $G$ is unbreakable and derive a contradiction. We recall the following result of Hayward [10].

Theorem 5. For every vertex $v$ of an unbreakable graph $G$ either:

(i) $v$ is contained in a hole in $G$, or

(ii) $v$ is contained in a hole in $\bar{G}$.

Note that the hypotheses on $G$ allow us to take $H$ a hole of size $|H| \geq 6$ containing $x$ the nose of a bull $\mathcal{B}(c, b x a, d)$. (See Fig. 1.) Let the vertices of the hole $H$ be labelled $v_{1}, v_{2}, \ldots, v_{n}$, with $v_{1}=x$, such that $v_{i}$ is adjacent to $v_{i+1}$, with indices taken modulo $n$, and $n=|H|$. We apply Lemma 5 (neighbourhood lemma revisited) to $H$ and some vertex $v$ of $\mathcal{B}(c, b x a, d)$.

To prove Theorem 3 , we distinguish a number of cases according to the other vertices $a, b, c, d$ of the bull $\mathcal{B}(c, b x a, d)$ being in the hole $H$ or not. We give below the complete list of the cases considered. In order to derive a contradiction in each case different tools are employed: reductions from one case to another, definition of a homogeneous set, or definition of a star cutset.

Case 1. Vertex a belongs to $H$.

Note that this implies that vertex $b$ does not belong to $H$. Assume $x=v_{1}$ and $a=v_{2}$. We further distinguish four subcases according to the adjacencies of $c$ and $d$ in $H$.

Case 1.1. Vertices $c, d$ do not belong to $H$.

This subcase is reduced to case 1.2 as follows. By Lemma 1, we have $b v_{n} \in E$ and vertex $b$ sees three consecutive vertices $v_{n}, v_{1}$ and $v_{2}$ of $H$. Now $\mathcal{B}\left(c, b x a, v_{3}\right)$ implies $c v_{3} \in E$. Now $x c, a c \notin E$ implies $c v_{n} \notin E$, as otherwise we have a $C_{5}$ 
in $G$. Now $\mathcal{B}\left(c, b x v_{n}, v_{n-1}\right)$ implies $c v_{n-1} \in E$. Finally, vertices $c, v_{3}, a, x, v_{n}$, $v_{n-1}$ induce a hole for case 1.2 .

Case 1.2. Vertex $c$ belongs to $H$, vertex $d$ does not belong to $H$.

Since $b$ sees edge $x a$ and vertex $c$ in $H$, Lemma 5 says $|H|=6$. Suppose first $b$ sees $x\left(=v_{1}\right), a\left(=v_{2}\right), v_{3}$ and $c\left(=v_{5}\right)$. Now Lemma 1 implies $a v_{6}$ or $b v_{6}$, and a contradiction. Hence we may assume that $b$ sees $v_{6}, x\left(=v_{1}\right), a\left(=v_{2}\right)$ and $c\left(=v_{4}\right)$.

Now a star cutset with center $b$ is found. Let $S$ be the subset of vertices $\left\{s: a, v_{6} \in N(s) ; d, v_{5}, c, v_{3} \notin N(s)\right\}$ of $G$. Let $S^{*}$ be the connected component of the subgraph of $G$ induced by $S$ containing $x$. If $b+N(b)-S^{*}$ is not a star cutset, then there exists $s \in S^{*}$ such that $s$ sees $z \notin\{b\} \cup N(b) \cup S^{*}$. Let $P$ be a shortest path from $b$ to $s$ in $S^{*}+b$. We argue by induction on the size of $P$ that no such vertex $s$ exists.

Let $P=b s$. Lemma 1 implies $z a$. If $z v_{6} \notin E$, then $\mathcal{B}\left(z, s v_{6} b, c\right)$ implies $z c \in E$, and now $\mathcal{B}\left(v_{6}, s a z, c\right)$ is a contradiction. So far we have: $z$ sees $a, v_{6}$; and $z$ misses $v_{3}, v_{5}$. It remains to show $z$ misses $d$ and $c$ to establish a contradiction. Lemma 5 says $d v_{3} \notin E$. If $z d \in E$, then $\mathcal{B}\left(v_{3}, a d z, v_{6}\right)$ implies $d v_{6} \in E$, and now $\mathcal{B}\left(c, b s v_{6}, d\right)$ is a contradiction. If $z c \in E$, then $\mathcal{B}(d, a s z, c)$ is a contradiction.

Let $P=b x s$. Note vertices $s, x, b, c$ induce a $P_{4}$, which implies $z c \notin E$. If $z a \notin E$, then $\mathcal{B}(d, a x s, z)$ implies $z d \in E$. Note $d v_{6} \notin E$, as $\mathcal{B}\left(d, v_{6} x b, c\right)$ is a contradiction. This in turn implies $d v_{5} \notin E$. Now $z v_{6} \in E$ gives the contradiction $\mathcal{B}\left(d, z s v_{6}, b\right)$, and $z v_{6}, z v_{5} \notin E$ imply $\mathcal{B}\left(z, s x v_{6}, v_{5}\right)$. Hence, $z a \notin E$ implies $z d, z v_{5} \in E$ and now vertices $d, a, b, c, v_{5}, z$ induce a $C_{6}$ which together with $x$ is a cap and a contradiction. If $z v_{6} \notin E$, then $\mathcal{B}\left(z, s x v_{6}, v_{5}\right)$ implies $z v_{5} \in E$, and now vertices $z, a, x, v_{6}, v_{5}$ induce a $C_{5}$ and a contradiction. Now Lemma 5 says if $z$ sees $a, v_{6}$ and $z$ misses $x, c$, then $z$ misses $v_{3}, v_{5}$. Finally, if $z d \in E$, then $\mathcal{B}\left(d, z s v_{6}, b\right)$ is a contradiction.

Let $P=b x x^{*} s$. If $z c \in E$, then vertices $c, b, x, x^{*}, s$ and $z$ induce a hole, with respect to which $N(a)$ contradicts Lemma 5. So, we have $z c \notin E$, which by the same argument implies $z v_{5}, z v_{3} \notin E$. If $z a \notin E$, then $\mathcal{B}\left(b, a x^{*} s, z\right)$ is a contradiction. If $z v_{6} \notin E$, then $\mathcal{B}\left(b, v_{6} x^{*} s, z\right)$ is a contradiction. If $z d \in E$, then $\mathcal{B}\left(d, z s v_{6}, b\right)$ is a contradiction. Therefore we have: $z$ sees $a, v_{6}$; and $z$ misses $c$, $d, v_{3}, v_{5}$ which establish a contradiction. Note the same argument applies to a longer path $P$.

Case 1.3. Vertex $d$ belongs to $H$, vertex $c$ does not belong to $H$.

Vertex $d=v_{3}$ and Lemma 1 implies $b v_{n}$. $\mathcal{B}\left(v_{n-1}, v_{n} x b, c\right)$ implies $c v_{n-1}$ or $c v_{n} \in E$. Suppose $c v_{n} \in E$. Now $\mathcal{B}\left(v_{n-1}, v_{n} c b, a\right)$ implies $c v_{n-1} \in E$, hence we conclude we must have $c v_{n-1} \in E$. Now suppose $c v_{n-1}, c v_{n} \in E$. Lemma 5 implies $c v_{n-2} \in E$. Now $\mathcal{B}\left(v_{n-2}, c v_{n} b, a\right)$ implies $b v_{n-2} \in E$, and $|H|=6$. But now $\mathcal{B}\left(d, v_{n-2} c b, x\right)$ gives a contradiction and we conclude that $c v_{n} \notin E$ and $c v_{n-1} \in E$. Now suppose $c v_{n-2} \in E$. Lemma 5 implies $|H|>6, b$ sees precisely $v_{n}, x=v_{1}, a=v_{2} ; c$ sees precisely $v_{n-1}, v_{n-2}, v_{n-3}$. But now $\mathcal{B}\left(b, c v_{n-2} v_{n-3}, v_{n-4}\right)$ gives a contradiction and we conclude that $c v_{n-2} \notin E$. Let $j$ be the smallest index such that $c v_{j} \in E$. The hole induced by $c, b, a, d, v_{4}, \ldots, v_{j}$, together with $x$ defines a cap unless $b$ 
is adjacent to a fourth vertex of $H$ which means $|H|=6$. Hence we may assume that $b$ sees $v_{6}, x=v_{1}, a=v_{2}$ and $v_{4}$, and that $d=v_{3}$.

Now, analogously to case 1.2, a star cutset with center $b$ is found as follows. Let $R$ be the subset of vertices $\left\{r: a, v_{6} \in N(r) ; d, v_{5}, c \notin N(r)\right\}$ of $G$. Let $R^{*}$ be the connected component of the subgraph of $G$ induced by $R$ containing $x$. If $b+N(b)-R^{*}$ is not a star cutset, then there exists $r \in R^{*}$ such that $r$ sees $z \notin\{b\} \cup N(b) \cup R^{*}$. Let $P$ be a shortest path from $b$ to $r$ in $R^{*}+b$. We argue by induction on the size of $P$ that no such vertex $r$ exists.

Let $P=b r$. Lemma 1 implies $z a$. If $z v_{6} \notin E$, then $\mathcal{B}\left(z, r v_{6} b, c\right)$ implies $z c \in E$, and now $\mathcal{B}\left(v_{6}, r a z, c\right)$ is a contradiction. So far we have: $z$ sees $a, v_{6}$; and $z$ misses $d, v_{5}$. It remains to show $z$ misses $c$ to establish a contradiction. If $z c \in E$, then $\mathcal{B}(c, z r a, d)$ is a contradiction.

Let $P=b x r$. Note vertices $r, x, b, c$ induce a $P_{4}$, which implies $z c \notin E$. If $z a \notin E$, then $\mathcal{B}(d, a x r, z)$ implies $z d \in E$, and now $z v_{6} \in E$ gives the contradiction $\mathcal{B}\left(d, z r v_{6}, b\right)$, and $z v_{6}, z v_{5} \notin E$ imply $\mathcal{B}\left(z, r x v_{6}, v_{5}\right)$. Hence, $z a \notin E$ implies $z v_{6} \notin E$ and $z d, z v_{5} \in E$. Now vertices $d, a, b, c, v_{5}, z$ induce a $C_{6}$ which together with $x$ is a cap and a contradiction. If $z v_{6} \notin E$, then $\mathcal{B}\left(z, r x v_{6}, v_{5}\right)$ implies $z v_{5} \in E$, and now vertices $a, x, v_{6}, v_{5}, z$ induce a $C_{5}$, a contradiction. If $z d \in E$, then $\mathcal{B}\left(d, z r v_{6}, b\right)$ is a contradiction. If $z v_{5} \in E$, then $\mathcal{B}\left(b, a r z, v_{5}\right)$ is a contradiction. Therefore we have: $z$ sees $a, v_{6}$; and $z$ misses $c, d, v_{5}$.

Let $P=b x x^{*} r$. If $z c \in E$, then vertices $c, b, x, x^{*}, r$ and $z$ induce a hole, with respect to which $N(a)$ contradicts Lemma 5 . So, we have $z c \notin E$, which by the same argument implies $z v_{5} \notin E$. If $z a \notin E$, then $\mathcal{B}\left(b, a x^{*} r, z\right)$ is a contradiction. If $z v_{6} \notin E$, then $\mathcal{B}\left(b, v_{6} x^{*} r, z\right)$ is a contradiction. If $z d \in E$, then $\mathcal{B}\left(d, z r v_{6}, b\right)$ is a contradiction. Therefore we have: $z$ sees $a, v_{6}$; and $z$ misses $c, d, v_{5}$ which establish a contradiction. Note the same argument applies to a longer path $P$.

Case 1.4. Vertices $c$, $d$ belong to $H$.

Clearly the hypothesis lead to a contradiction as follows. Since $b$ sees edge $x a$ and vertex $c$ in $H$, Lemma 5 says $|H|=6$. Since $d$ is adjacent to $a$, we have $d=v_{3}$. Since $b$ is not adjacent to $d$, we have that $b$ sees $v_{6}, x=v_{1}, a=v_{2}$ and $c=v_{4}$. Now $c d \in E$ is a contradiction.

Case 2. Vertices $a, b$ do not belong to $H$.

The key property used in case 2 is: since $a, b$ do not belong to $H$, we may apply Lemma 1 to both $v_{2}$ and $v_{n}$, neighbours of nose $x$. Hence, vertex $v_{2}$ is adjacent to $a$ or $b$, and vertex $v_{n}$ is adjacent to $a$ or $b$. This in turn implies that vertex $a$ or vertex $b$ see three consecutive vertices of hole $H$. We further distinguish three subcases according to the adjacencies of $c$ and $d$ in $H$. Note that in case 1 , we did not have symmetry in $a$ and $b$. For the present case, we have just three subcases as we have symmetry in $a$ and $b$.

Case 2.1. Vertices $c, d$ do not belong to $H$.

Suppose $a$ sees $v_{n}$ but not $v_{2}$, and $b$ sees $v_{2}$ but not $v_{n}$. Either we get a different hole for case 1 by replacing a vertex of $H$ by $a$ or $b$; or both $a$ and $b$ see a fourth vertex in $H,|H|=6$, and $\mathcal{B}\left(v_{n}, a b v_{3}, v_{4}\right)$ is a contradiction. Hence, we may 
assume $a$ sees $v_{n}$ and $v_{2}$. Suppose $d$ misses $v_{n}$ and $v_{2}$. Hence $\mathcal{B}\left(d, a x v_{n}, v_{n-1}\right)$, $\mathcal{B}\left(d, a x v_{2}, v_{3}\right)$ imply $d v_{n-1}, d v_{3} \in E$. Now vertices $d, v_{n-1}, v_{n}, x, v_{2}, v_{3}$ induce a $C_{6}$ for case 2.2 . Now, by symmetry, we assume $d$ sees $v_{n}$. $\mathcal{B}\left(d, a x v_{2}, v_{3}\right)$ implies $d v_{2}$ or $d v_{3}$. Now we must have $d v_{2} \in E$, as otherwise, vertices $d, v_{n}, x, v_{2}$, $v_{3}$ induce a $C_{5}$, a contradiction. Now Lemma 5 says $d$ misses $v_{n-1}$ or $v_{3}$, by symmetry we may assume $d$ misses $v_{n-1}$. Now $\mathcal{B}\left(v_{n-1}, v_{n} a d, v_{3}\right)$ says $d$ also misses $v_{3}$. Now $b v_{n-1} \notin E$, as otherwise $\mathcal{B}\left(v_{n-1}, b x a, d\right)$ is a contradiction. Now $b v_{n} \in E$, as otherwise $\mathcal{B}\left(v_{n-1}, v_{n} d a, b\right)$ is a contradiction. Now Lemma 5 says $b v_{2} \in E$, $b v_{3} \notin E$. Analogously, $c v_{n}, c v_{2} \in E ; c v_{n-1}, c v_{3} \notin E$.

Consider the set $B=\left\{s: s v_{n}, s v_{2} \in E ; s z \notin E, z \in H-\left\{x, v_{n}, v_{2}\right\}\right\}$. We shall exhibit a homogeneous set $B^{\prime}$ contained in $B$ which establishes a contradiction.

First, note that $B$ contains vertices $x, a, b, c, d$. We have shown that $x, a, b, c, d$ see $v_{n}, v_{2}$, miss $v_{n-1}, v_{3}$. We have to show that $x, a, b, c, d$ miss $v_{j}, 4 \leq j \leq n-2$. This clearly holds for $x$. Vertices $a$ and $b$ see three consecutive vertices in $H: v_{n}$, $x$ and $v_{2}$. In case $a$ or $b$ see another vertex of $H$, we have $H=C_{6}$ and this extra vertex is $v_{4}$. Now $a v_{4}$ and $\mathcal{B}\left(v_{4}, a x v_{2}, c\right)$ imply $c v_{4} ; b v_{4}$ and $\mathcal{B}\left(v_{4}, b x v_{6}, d\right)$ imply $d v_{4}$. Now $c v_{4}$ implies $b v_{4} \notin E$, as otherwise $\mathcal{B}\left(v_{3}, v_{4} c b, x\right)$ is a contradiction; $d v_{4}$ implies $a v_{4} \notin E$, as otherwise $\mathcal{B}\left(v_{5}, v_{4} d a, x\right)$ is a contradiction. Suppose $b v_{4} \in E$. This implies $H=C_{6}$ and $d v_{4} \in E, a v_{4} \notin E, c v_{4} \notin E$. Now $\mathcal{B}\left(v_{4}, d a v_{6}, c\right)$ is a contradiction, and we may conclude: $b v_{4} \notin E$, and symmetrically $a v_{4} \notin E$. Suppose $c v_{j} \in E, 4 \leq j \leq n-2$. This implies $d v_{j} \notin E$. Now $\mathcal{B}\left(v_{j}, c b v_{n}, d\right)$ is a contradiction and we may conclude $c v_{j} \notin E$, and symmetrically $d v_{j} \notin E$, $4 \leq j \leq n-2$.

Let $B^{\prime}$ be a maximal subset of $B$ containing vertices $x, a, b, c, d$ such that the subgraphs induced by $B^{\prime}$ in $G$ and in $\bar{G}$ are connected. If $B^{\prime}$ is not a homogeneous set, there exists $w \in V \backslash B^{\prime}$ which disagrees on $B^{\prime}$, i.e., $w$ is such that $w$ has a neighbour in $B^{\prime}$ and a nonneighbour in $B^{\prime}$. Note that such a vertex $w$ must in fact belong to $V \backslash B$ by the maximality property satisfied by $B^{\prime}$. In addition, $w$ must disagree on an edge $b_{1} b_{2} \in E$, with $b_{1}, b_{2} \in B^{\prime}$. For let $b^{\prime}, b^{\prime \prime}$ be such that $w b^{\prime} \in E$, $w b^{\prime \prime} \notin E$. Since $B^{\prime}$ is connected, take a $b^{\prime} b^{\prime \prime}$-path $P$ inside $B^{\prime}$ and consider the first $b^{*} \in P$ such that $w b^{*} \notin E$. Similarly, $w$ must disagree on a non edge $b_{1} b_{2} \notin E$, with $b_{1}, b_{2} \in B^{\prime}$.

Suppose $w$ sees $v_{n}$ and misses $v_{2}$. Let $u, v \in B^{\prime}$ be such that $w u \in E, w v \notin E$. Consider two holes: $H_{1}$ induced by $v_{n-1}, v_{n}, u, v_{2}, v_{3}, \ldots, v_{n-2} ; H_{2}$ induced by $v_{n-1}, v_{n}, v, v_{2}, v_{3}, \ldots, v_{n-2}$ and the neighbourhood of $w$ with respect to these two holes. Since $w$ is adjacent to $u$ and $v_{n}$, by considering $H_{1}$ we have $w v_{n-1} \in E$. Now considering $H_{2}$ and that $w$ sees $v_{n}, v_{n-1}$ and misses $v$, we have $w v_{n-2} \in E$, and the neighbourhood of $w$ with respect to $H_{1}$ is a contradiction.

Suppose $w$ sees $v_{n}$ and $v_{2}$. Let $r$ be a vertex of $B^{\prime}$ such that $r w \in E$. Consider the hole $H_{r}$ induced by $v_{n-1}, v_{n}, r, v_{2}, v_{3}, \ldots, v_{n-2}$ and the neighbourhood of $w$ with respect to $H_{r}$. We have that $w$ misses both $v_{n-1}$ and $v_{3}$. Now $w$ has to see a vertex $z \in H \backslash\left\{v_{n-1}, v_{n}, x, v_{2}, v_{3}\right\}$ as otherwise $w \in B$. Now consider a non edge $u v \notin E$, with $u, v \in B^{\prime}$ with respect to which $w$ disagrees: $w u \in E, w v \notin E$. Now $\mathcal{B}\left(z, w u v_{n}, v\right), \mathcal{B}\left(z, w u v_{2}, v\right)$ are contradictions. 
Finally suppose $w$ misses $v_{n}$ and $v_{2}$. Let $u v \in E\left(u, v \in B^{\prime}\right)$, be an edge with respect to which $w$ disagrees: $w u \in E, w v \notin E$. The contradiction $\mathcal{B}\left(v_{n-1}, v_{n} v u, w\right)$ implies $w v_{n-1} \in E$, and the contradiction $\mathcal{B}\left(v_{3}, v_{2} v u, w\right)$ implies $w v_{3} \in E$. By the maximality condition on $B^{\prime}$, we may consider $\pi$ a total order on $B^{\prime}$ with respect to which the vertices of $B$ are selected to be in $B^{\prime}$ as follows: $\pi=d, a, b, c, x, b_{1}, b_{2}, \ldots$, such that each subgraph $G_{i}$ induced by $d, a, b, c, x, b_{1}, b_{2}, \ldots, b_{i}$ in $G$ is connected and has its complement connected. Now with respect to $\pi$, let $u$ be the first vertex such that $w$ sees all vertices before $u$, and $w$ misses $u$. In case $u$ is equal to or greater than $b$, there is an edge $z z^{\prime} \in E$, such that $z, z^{\prime}$ are before $u$, on which $u$ disagrees. Now $\mathcal{B}\left(v_{n-1}, w z z^{\prime}, u\right), \mathcal{B}\left(v_{3}, w z z^{\prime}, u\right)$ are contradictions. In case $u=a$, the contradiction $\mathcal{B}\left(w, d u v_{n}, c\right)$ implies $w c \in E$ which in turn implies $w b \in E$. Now $\mathcal{B}\left(v_{n-1}, w c b, a\right)$ and $\mathcal{B}\left(v_{3}, w c b, a\right)$ are contradictions. In case $u=d$, if $w$ misses $b$ and sees $c$, then $\mathcal{B}\left(w, c b v_{2}, d\right)$ is a contradiction. If $w$ misses $c$ and sees $b$, then $\mathcal{B}\left(w, b c v_{2}, d\right)$ is a contradiction. If $w$ sees $b$ and $c$, and misses $a$, then $\mathcal{B}\left(v_{n-1}, w c b, a\right)$ is a contradiction. If $w$ sees $a$, and misses $b$ and $c$, then $\mathcal{B}\left(w, a d v_{2}, c\right)$ is a contradiction. If $w$ sees $a$ and $b$, then $\mathcal{B}\left(v_{n-1}, w b a, d\right)$ is a contradiction. If $w$ misses $a, b$ and $c$, let $u^{*}$ be the first vertex seen by $w$, and let $e, h$ be two nonadjacent vertices before $u^{*}$ such that $u^{*} e \notin E, u^{*} h \in E$, to get the final contradiction $\mathcal{B}\left(w, u^{*} h v_{2}, e\right)$.

Case 2.2. Vertex $c$ belongs to $H$, vertex d does not belong to $H$.

This subcase is first reduced to $|H|=6$, with $a$ or $b$ seeing precisely four vertices of $H$ as in Figure 2 as follows. We know that $a$ or $b$ see three consecutive vertices of $H$. Suppose $b$ sees three consecutive vertices of $H$. Either $b$ sees precisely four vertices of $H$ and $|H|=6$, or $b$ sees precisely three consecutive vertices of $H$. Now, in the latter case, since $c, x \in H$, and $x=v_{1}$, we may assume $b$ sees precisely $x\left(=v_{1}\right), v_{2}$ and $c\left(=v_{3}\right)$ in $H$, which by replacing $v_{2}$ by $b$ in $H$, gives a hole $H^{\prime}$ for case 1 . Now suppose that $a$ sees three consecutive vertices of $H$ but $b$ does not see three consecutive vertices of $H$. Either $a$ sees precisely four vertices of $H$ and $|H|=6$, or $a$ sees precisely three consecutive vertices of $H$. In the latter case, either we get a hole $H^{\prime}$ for case 1 by replacing a vertex of $H$ by $a$, or $a$ sees precisely $v_{n}, x\left(=v_{1}\right)$ and $v_{2}$. In addition, $b$ sees $c$ in $H$. So either we get a different hole for case 1 by replacing a vertex of $H$ by $b$, or $b$ misses $v_{n}$ and $v_{2}$, and $b$ sees $v_{3}$ and $v_{n-1}$. Now $\mathcal{B}\left(v_{n-1}, b x a, d\right)$ implies $d v_{n-1} \in E$, and $\mathcal{B}\left(v_{3}, b x a, d\right)$ implies $d v_{3} \in E$, which in turn imply $d v_{n}, d v_{2} \notin E$. Now vertices $d, v_{n-1}, v_{n}$, $x, v_{2}, v_{3}$ induce a $C_{6}$, with respect to which $a$ sees precisely four vertices: $v_{6}$, $x\left(=v_{1}\right), v_{2}$ and $d\left(=v_{4}\right)$, as required. Note that this latter $C_{6}$ contains $x$ and $d$, and does not contain $a, b$, and $c$. Note further that the neighbourhood of $a$ with respect to this $C_{6}$ says it corresponds to the third subcase considered below. Now we consider four subcases.

- Vertex $b$ sees precisely four vertices: $v_{3}, v_{4}, v_{5}$ and $x\left(=v_{1}\right)$ in $H$.

This implies that $a$ sees $v_{2}$ and $v_{6}$, and misses $v_{3}$ and $v_{5}$. Suppose $a$ sees $v_{4}$. This implies $c \neq v_{4}$ and we may assume $c=v_{3}$. Now $\mathcal{B}\left(d, a x b, v_{5}\right)$ implies $d v_{5} \in E$ and $\mathcal{B}\left(v_{3}, v_{2} x a, d\right)$ implies $v_{2} d \in E$, which in turn implies that $d$ sees $v_{4}, v_{5}, v_{6}$ and $v_{2}$ in $H$. Now $\mathcal{B}\left(c, v_{2} a d, v_{5}\right)$ is a contradiction and we conclude that $a$ misses $v_{4}$. If $d$ 
disagrees on $v_{3}, v_{4}, v_{5}$ then the set $\left\{v_{3}, v_{4}, v_{5}, b, x, d\right\}$ contains a bull: either $d$ sees $v_{4}$, which implies $d$ misses $v_{3}$ or $v_{5}$, which implies a bull on vertices $d, v_{4}, b, x$ and vertex $v_{3}$ or $v_{5}$, or $d$ misses $v_{4}$, which implies $d$ sees $v_{3}$ or $v_{5}$, which again implies a bull on vertices $d, v_{4}, b, x$ and vertex $v_{3}$ or $v_{5}$. So $d$ agrees on $v_{3}, v_{4}, v_{5}$ and since $d$ misses $c$, we conclude that $d$ also misses $v_{3}, v_{4}$, and $v_{5}$. But now vertices $d, b, a$, $x$ and any choice of a fifth vertex chosen in $\left\{v_{3}, v_{4}, v_{5}\right\}$ give a contradiction.

- Vertex $b$ sees precisely four vertices: $v_{3}, v_{5}, v_{6}$ and $x\left(=v_{1}\right)$ in $H$.

Note that $c \in\left\{v_{3}, v_{5}\right\}$. Lemma 1 says $a v_{2} \in E$, which implies $a v_{3} \in E$ or $a v_{6} \in E$. Suppose $a v_{3} \in E$. Either we have $x, a$ on a $C_{6}$ for case 1 , or we have $a v_{5} \in E$ which contradicts $c \in H$. We conclude that $a$ misses $v_{3}$ and $v_{5}$, and $a$ sees $v_{6}$, Now $\mathcal{B}\left(d, a x v_{2}, v_{3}\right)$ implies $d v_{2} \in E$ or $d v_{3} \in E$, and $\mathcal{B}\left(d, a x v_{6}, v_{5}\right)$ implies $d v_{6} \in E$ or $d v_{5} \in E$. Suppose $d v_{3} \in E$. This implies $c=v_{5}$ and that $d$ misses $v_{5}$, which in turn implies $d v_{6} \in E$. Since $d$ sees $v_{3}$ and $v_{6}$, and misses $v_{1}$ and $v_{5}$, we have that $d$ also sees both $v_{2}$ and $v_{4}$. Now $\mathcal{B}\left(c, v_{4} v_{3} d, a\right)$ implies $a v_{4} \in E$, and the contradiction $\mathcal{B}\left(x, a d v_{4}, c\right)$. We conclude that $d$ misses $v_{3}$ and that $d$ sees $v_{2}$. Since $d$ has to see $v_{5}$ or $v_{6}$, and vertices $v_{2}$ and $v_{5}$ are antipodals with respect to $H$, we have that $d$ sees $v_{6}$ and $\mathcal{B}\left(d, v_{6} x b, v_{3}\right)$ is now a contradiction.

- Vertex $b$ sees precisely four vertices: $v_{2}, v_{4}, v_{6}$ and $x=v_{1}$ in $H$.

Note that $c=v_{4}$. If $d v_{6} \in E$, then $\mathcal{B}\left(c, b x v_{6}, d\right)$ is a contradiction, and similarly if $d v_{2} \in E$. So $d v_{6} \notin E, d v_{2} \notin E$. In addition, since both $v_{3}$ and $v_{5}$ are adjacent to $c$, we have $d v_{5} \in E$ implies $a v_{5} \in E$, and $d v_{3} \in E$ implies $a v_{3} \in E$. Now the contradictions $\mathcal{B}\left(c, v_{5} d a, x\right)$ and $\mathcal{B}\left(c, v_{3} d a, x\right)$ say $d v_{3} \notin E, d v_{5} \notin E$. In particular, the conclusion is that $d$ does not see $H$. Now, if $a v_{6} \in E$, then $a v_{2} \in E$ or $a v_{5} \in E$. If $a v_{5} \in E$, then $\mathcal{B}\left(c, v_{5} v_{6} a, d\right)$ implies $d v_{5} \in E$, which is a contradiction. So $a v_{5} \notin E$, and now $\mathcal{B}\left(v_{5}, v_{6} x a, d\right)$ is a contradiction. So $a v_{6} \notin E$, and symmetrically, $a v_{2} \notin E$.

We shall find a star cutset as follows. Let $S$ be the set of vertices $\left\{s: a, v_{2}, v_{6} \in\right.$ $\left.N(s) ; d, v_{5}, c, v_{3} \notin N(s)\right\}$ of $G$. Let $S^{*}$ be the connected component of the subgraph of $G$ induced by $S$ containing $x$. If $b+N(b)-S^{*}$ is not a star cutset, then there exists $s \in S^{*}$ such that $s$ sees $z \notin\{b\} \cup N(b) \cup S^{*}$. Let $P$ be a shortest path from $b$ to $s$ in $S^{*}+b$. We argue by induction on the size of $P$ that no such vertex $s$ exists.

Let $P=b s$. Lemma 1 implies $z a \in E$. Suppose $z v_{6} \notin E$. Then $\mathcal{B}\left(c, b v_{6} s, z\right)$ implies $z c \in E$ which implies $\mathcal{B}\left(c, z a x, v_{6}\right)$ is a contradiction. So $z v_{6} \in E$, and symmetrically $z v_{2} \in E$, which implies $z v_{5} \notin E, z v_{3} \notin E$. If $z d \in E$, then $\mathcal{B}\left(d, z x v_{6}, v_{5}\right)$ is a contradiction. Now, if $z c \in E$, then $\mathcal{B}(d, a x z, c)$ is a contradiction.

Let $P=b x s$. Note that vertices $c, b, x, s$ induce a $P_{4}$ which implies $z c \notin E$. Now $z a \notin E$ or $\mathcal{B}(z, a x b, c)$ is a contradiction. Now $z d \in E$ or $\mathcal{B}(d, a x s, z)$ is a contradiction. Now $z v_{6} \notin E$ or vertices $z, v_{6}, x, a, d$ induce a $C_{5}$, and $z v_{2} \notin E$ or vertices $z, v_{2}, x, a, d$ induce a $C_{5}$. Now $z v_{5} \in E$ or $\mathcal{B}\left(v_{5}, v_{6} x s, z\right)$ is a contradiction, and $z v_{3} \in E$ or $\mathcal{B}\left(v_{3}, v_{2} x s, z\right)$ is a contradiction. Now vertices $c, b, x, s, z, v_{5}$ induce a $C_{6}$ through $x, b$ for case 1 .

Let $P=b x x^{*} s . \mathcal{B}(c, b x a, s)$ is a contradiction and note the same argument applies to a longer path $P$. 
- Vertex a sees precisely four vertices in $H$ but $b$ does not see precisely four vertices in $H$.

Since $b$ is adjacent to $x$ and $c$, two non consecutive vertices of $H$, the hypothesis implies that $x$ and $c$ are not antipodals with respect to $H$. In addition, vertex $c$ is not adjacent to $a$. So we may assume $x=v_{1}, c=v_{5}$, and that vertex $a$ sees precisely $v_{2}, v_{4}, v_{6}$ and $x\left(=v_{1}\right)$. Now $\mathcal{B}\left(c, v_{6} x a, d\right)$ implies $v_{6} d \in E$ and $\mathcal{B}\left(c, v_{6} d a, v_{2}\right)$ implies $d v_{2} \in E$. Now $b$ sees $v_{3}$, as otherwise vertices $c, b, x, v_{2}, v_{3}, v_{4}$ induce a $C_{6}$ for case 1 . Now $b$ misses $v_{6}$, as otherwise $b$ sees four vertices in $H$. Now $\mathcal{B}\left(b, v_{3} v_{2} d, v_{6}\right)$ implies $d v_{3} \notin E$ and finally $\mathcal{B}\left(v_{3}, b x a, d\right)$ is a contradiction.

Case 2.3. Vertices $c$, $d$ belong to $H$.

Without loss of generality, suppose $a$ sees three consecutive vertices of hole $H$. If $a$ sees $v_{n}, x\left(=v_{1}\right)$ and $v_{2}$, then Lemma 5 says $d=v_{j}$, with $j \neq 1,2,3, n-1, n$; and $|H|=6$. Now this contradicts $c \in H$. Otherwise, we have $a$ sees $x\left(=v_{1}\right), v_{2}$ and $v_{3} ; b$ sees $v_{n-1}, v_{n}$, and $x\left(=v_{1}\right)$. This implies $c=v_{n-1}$ and $d=v_{3}$, vertex $b$ sees precisely three consecutive vertices: $c\left(=v_{n-1}\right), v_{n}$, and $x\left(=v_{1}\right)$; vertex $a$ sees precisely three consecutive vertices: $x\left(=v_{1}\right), v_{2}, v_{3}(=d)$ in $H$. Now we have the bull $\mathcal{B}\left(v_{n-2}, c v_{n} b, a\right)$ intersecting $\mathcal{B}(c, b x a, d)$, and a contradiction.

\section{ReCOGNition ALGORIthM}

The proposed polynomial-time algorithm for recognizing bull-reducible Berge graphs is based on the following decomposition by homogeneous sets:

Fact 2. If $G$ contains a homogeneous set $O$ and $h$ is any vertex of $O$, then $G$ is Berge if and only if $G-(O-h)$ and the subgraph induced by $O$ are Berge.

A simple proof of Fact 2 follows from the definitions of homogeneous sets and Berge graphs. Lovász [14] has shown in his proof of the Perfect Graph Theorem that $G$ is perfect if and only if $G-(O-h)$ and the subgraph induced by $O$ are perfect. In a homogeneous set decomposition tree each internal node corresponds to a graph $G$ containing a homogeneous set $O$ and has two children corresponding to $G-(O-h)$ and to the subgraph induced by $O$.

Fact 3. A homogeneous set decomposition tree rooted on a graph $G(V, E)$ with $|V|=n$ has $\mathcal{O}(n)$ nodes.

A simple proof of Fact 3 uses that if $G(V, E)$ with $|V|=n$ is an internal node, then its children $G_{1}\left(V_{1}, E_{1}\right)=G-(O-h)$ and $G_{2}\left(V_{2}, E_{2}\right)$, the subgraph induced by $O$, satisfy: $\left|V_{1}\right|+\left|V_{2}\right|=n+1$ and is presented in [16] where a homogeneous set decomposition tree is also built, this time for the recognition of bull-free Berge graphs.

The proposed recognition algorithm reduces in polynomial time the recognitionof bull-reducible Berge graphs to the recognition of bull-free Berge graphs by 
performing another reduction operation which also preserves the property of being Berge:

Lemma 6. Let $G$ be a $C_{5}$-free, bull-reducible and homogeneous set-free graph. Let $x$ be the nose of a bull in $G$. Then $G-x$ is odd hole free if and only if $G$ is odd hole free.

Proof. Let $H$ be an odd hole in $G$ containing $x$, the nose of the bull $\mathcal{B}(c, b x a, d)$. Let the vertices of the hole $H$ be labelled $v_{1}, v_{2}, \ldots, v_{n}$, with $x=v_{1}$, such that $v_{i}$ is adjacent to $v_{i+1}$, with indices taken modulo $n$, and $n=|H|$. We distinguish two cases:

Case 6.1. Neither a nor b belong to $H$.

If $b$ sees both $v_{2}$ and $v_{n}$, then by Lemma 5 (neighbourhood lemma revisited) $b$ and $x$ are twins with respect to $H$. Hence we have another hole $H^{\prime}=H-x+b$ induced by $b, v_{2}, \ldots, v_{n}$ in $G-x$, as required. Similarly if $a$ sees both $v_{2}$ and $v_{n}$.

By Lemma 1 (Domination Lemma) $N(x) \subseteq N(a) \cup N(b)$, which implies $v_{2}$, $v_{n} \in N(a) \cup N(b)$. We may assume the edges $b v_{n}, a v_{2}$. Now $x$ sees four consecutive vertices $v_{n}, b, a, v_{2}$ on the cycle induced by $v_{2}, v_{3}, \ldots, v_{n-1}, v_{n}, b, a$ which implies that $a$ or $b$ see more vertices of the hole $H$. Without loss of generality assume $a$ sees another vertex of $H$ besides $x$ and $v_{2}$. Lemma 5 implies that $a$ sees precisely $x=v_{1}, v_{2}$ and $v_{3}$. Now we have a bull $\mathcal{B}\left(b, a v_{2} v_{3}, v_{4}\right)$ and a contradiction.

Case 6.2. a belongs to $H$.

Note that this implies $b$ does not belong to $H$. Say $a=v_{2}$. Now, $v_{n} \in N(x)$ and Lemma 1 imply $v_{n} \in N(a) \cup N(b)$, which in turn implies edge $v_{n} b$. Again, by Lemma 5 , we have another hole $H^{\prime \prime}=H-x+b$ induced by $b, v_{2}, \ldots, v_{n}$ in $G-x$, as required.

The analysis of these two cases proves Lemma 6.

Theorem 6. Algorithm 1 correctly identifies in polynomial time whether $G$ is a bull-reducible Berge graph.

Proof. Algorithm 1 builds a decomposition tree whose internal nodes correspond to graphs having a homogeneous set, while the leaves correspond to indecomposable graphs, i.e., graphs with no homogeneous set. By Lemma 6, we know that every time Algorithm 1 removes a nose $x$ from a graph $F$, we have $F-x$ is odd hole free if and only if $F$ is odd hole free. The properties of being $C_{5}$-free, bull-reducible, homogeneous set-free, and $x$ being the nose of a bull are satisfied by $F$ if and only if they are also satisfied by $\bar{F}$. Hence we actually have: $\bar{F}-x$ is odd hole free if and only if $\bar{F}$ is odd hole free, and therefore: $F-x$ is Berge if and only if $F$ is Berge. So this property and Fact 2 imply that the input graph $G$ is Berge if and only if the indecomposable graphs in $\mathcal{L}_{2}$ are Berge. This proves that Algorithm 1 correctly reduces the recognition of bull-reducible Berge graphs to the recognition of bull-free Berge graphs.

Algorithm 1 runs in polynomial time because its complexity is dominated by the complexity of an Algorithm 2 for bull-free Berge graph recognition multiplied by 
Algorithm 1. Recognizing bull-reducible Berge graphs.

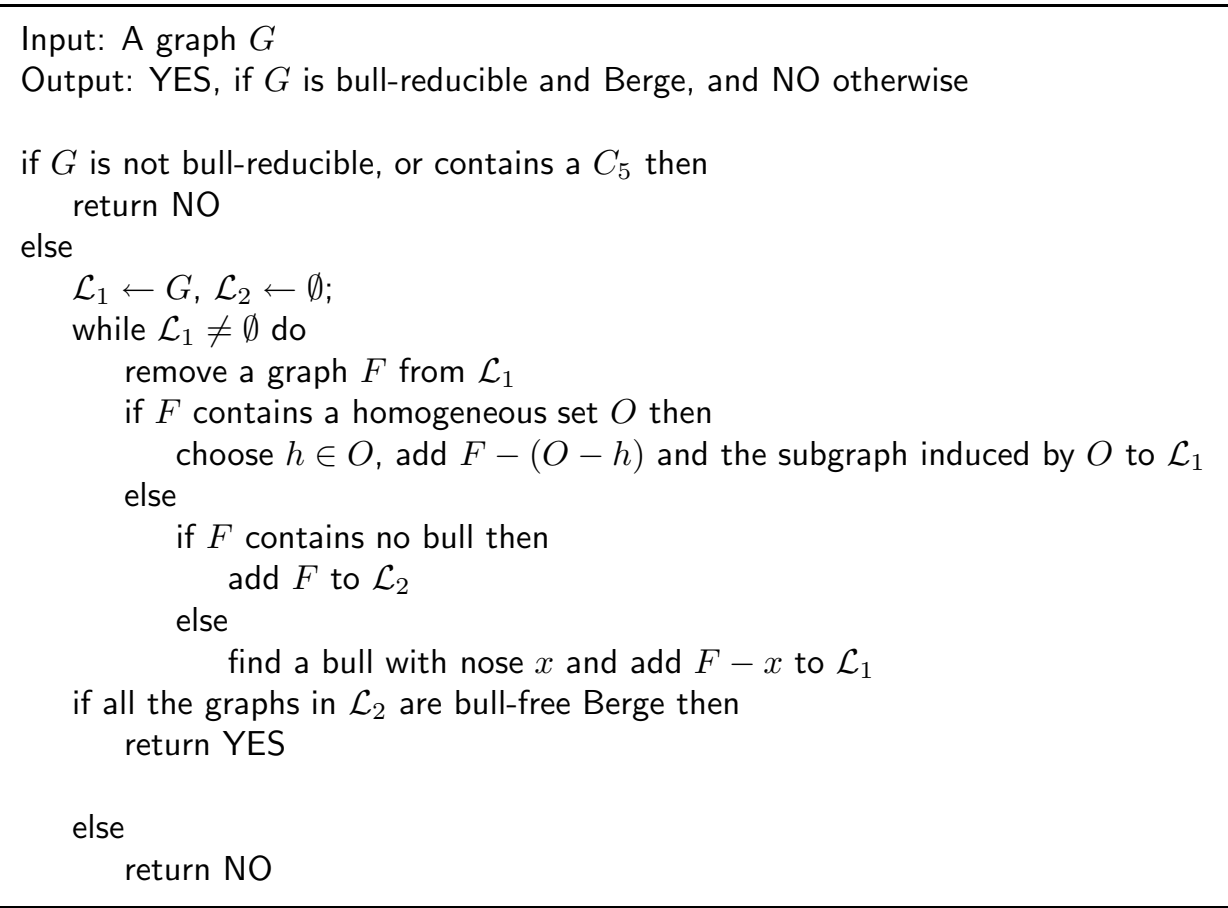

the number of calls for this Algorithm 2. In [16], bull-free Berge graph recognition is done in time $\mathcal{O}\left(n^{5}\right)$. Fact 3 implies that the number of calls for bull-free Berge graph recognition is $\mathcal{O}(n)$. Therefore Algorithm 1 runs in time $\mathcal{O}\left(n^{6}\right)$.

\section{Open PROBlems}

Several interesting open problems remain: Can we find a more efficient recognition algorithm? Can we find a decomposition theorem for this class of Berge graphs? Can we find efficient algorithms for the optimization problems clique number and minimum coloring for this new class of perfect graphs, or even for the more restricted class of bull-free perfect graphs?

The proposed proof of the Strong Perfect Graph Conjecture [4] still leaves a lot of questions open. Since the proposed decomposition obtained in the proof does not imply a nice composition procedure by which we can build all perfect graphs starting from the basic classes (because the skew cutset decomposition is not perfection preserving), it is still worth to continue trying to understand perfect graphs through different other means. Polynomial-time algorithms to recognize Berge graphs have subsequently been announced $[3,5,13]$. Polynomial-time 
combinatorial algorithms for the four optimization problems, based on a structural analysis and decomposition of perfect graphs are still being sought.

\section{REFERENCES}

[1] C. Berge and V. Chvátal, Topics on Perfect Graphs. North-Holland, Amsterdam, Ann. Discrete Math. 21 (1984).

[2] V. Chvátal, Star-cutsets and perfect graphs. J. Combin. Theory Ser. B 39 (1985) 189-199.

[3] M. Chudnovsky, G. Cornuejols, X. Liu, P. Seymour and K. Vuskovic, Cleaning for Bergeness, manuscript (2003).

[4] M. Chudnovsky, N. Robertson, P. Seymour and R. Thomas, The Strong Perfect Graph Theorem, manuscript (2003).

[5] M. Chudnovsky and P. Seymour, Recognizing Berge graphs, manuscript (2003).

[6] V. Chvátal and N. Sbihi, Bull-free Berge graphs are perfect. Graphs Combin. 3 (1987) $127-139$.

[7] C.M.H. de Figueiredo, F. Maffray and O. Porto, On the structure of bull-free perfect graphs. Graphs Combin. 13 (1997) 31-55.

[8] C.M.H. de Figueiredo, F. Maffray and O. Porto, On the structure of bull-free perfect graphs, 2: The weakly chordal case. Graphs Combin. 17 (2001) 435-456.

[9] M. Grötschel, L. Lovász and A. Schrijver, Polynomial algorithms for perfect graphs, in Topics on Perfect Graphs, edited by C. Berge and V. Chvátal. North-Holland, Amsterdam, Ann. Discrete Math. 21 (1984) 325-356.

[10] R.B. Hayward, Discs in unbreakable graphs. Graphs Combin. 11 (1995) 249-254.

[11] R.B. Hayward, Bull-free weakly chordal perfectly orderable graphs. Graphs Combin. 17 (2001) 479-500.

[12] B. Jamison and S. Olariu, $P_{4}$-reducible graphs - a class of uniquely tree-representable graphs. Stud. Appl. Math. 81 (1989) 79-87.

[13] X. Liu, G. Cornuejols and K. Vuskovic, A polynomial algorithm for recognizing perfect graphs, manuscript (2003).

[14] L. Lovász, Normal hypergraphs and the weak perfect graph conjecture. Discrete Math. 2 (1972) 253-267.

[15] J.L. Ramirez-Alfonsin and B.A. Reed, Perfect Graphs. Wiley (2001).

[16] B. Reed and N. Sbihi, Recognizing bull-free perfect graphs. Graphs Combin. 11 (1995) 171-178. 